EPJ manuscript No.

(will be inserted by the editor)

\title{
Steady-State Dynamics of the Forest Fire Model on Complex Networks
}

\author{
Jean-Daniel Bancal ${ }^{1}$ and Romualdo Pastor-Satorras ${ }^{2}$ \\ ' 1 Institut de Théorie des Phénomènes Physiques, Ecole Polytechnique Fédérale de Lausanne, CH-1015 Lausanne, Switzerland \\ e-mail: jean-daniel.bancal@unige.ch \\ 2 Departament de Física i Enginyeria Nuclear, Universitat Politécnica de Catalunya, Campus Nord, 08034 Barcelona, Spain
}

November 1, 2018

\begin{abstract}
Many sociological networks, as well as biological and technological ones, can be represented in terms of complex networks with a heterogeneous connectivity pattern. Dynamical processes taking place on top of them can be very much influenced by this topological fact. In this paper we consider a paradigmatic model of non-equilibrium dynamics, namely the forest fire model, whose relevance lies in its capacity to represent several epidemic processes in a general parametrization. We study the behavior of this model in complex networks by developing the corresponding heterogeneous mean-field theory and solving it in its steady state. We provide exact and approximate expressions for homogeneous networks and several instances of heterogeneous networks. A comparison of our analytical results with extensive numerical simulations allows to draw the region of the parameter space in which heterogeneous mean-field theory provides an accurate description of the dynamics, and enlights the limits of validity of the mean-field theory in situations where dynamical correlations become important.
\end{abstract}

Key words. Complex networks - Forest Fire - Epidemics - Mean Field Theory

PACS. PACS-key discribing text of that key - 89.75.-k Complex systems - 64.60.aq Networks

\section{Introduction}

' The heterogeneous topology of a networked substrate has been proven to have a large impact on dynamical processes taking place on top of it 12 . These topological effects are especially remarkable in the case of scale-free (SF) complex networks 34, characterized by a degree distribution $P(k)$, defined as the probability that an element in the network (vertex) is connected to $k$ other elements, 'that exhibits a power-law behavior, $P(k) \sim k^{-2-\gamma}$, with $0<\gamma \leq 1$ 1 . The diverging second moment $\left\langle k^{2}\right\rangle$ of the degree distribution has thus been found to be at the core of the peculiar behavior observed in a wide array of nonequilibrium dynamical processes, ranging from percolation [56], absorbing-state phase transitions [7, self-organized criticality 89], synchronization phenomena [10, opinion dynamics [11, etc.

The interplay between topology and dynamics has been particularly studied in the case of epidemic processes [12, where the relevant substrate is the network of contacts through which the disease spreads [13. Starting from the first observations of an epidemic threshold scaling as the inverse of the second moment of the degree distribution,

1 To ease the notation in our mathematical treatment, we will use this definition of the degree exponent in a power-law degree distribution. and thus vanishing in the thermodynamic limit of an infinite network size [14151617], a wealth of interesting and relevant results have arisen, dealing, to mention just a few, with immunization strategies 1819, effects of bipartite (heterosexual) populations [20] or epidemic forecasting [21].

The understanding of the features of epidemic spreading is mainly based on the analysis of compartmental models [22, in which the population is divided into different classes, according to the stage of the disease. Individuals (the vertices in the network) are in this way classified as susceptible (healthy and capable to contract the disease), infected (sick, and capable to transmit the disease), recovered (immunized or dead), etc. With these definitions, different epidemic models can be formulated, according to the succession of states that the evolution of the disease imposes on each individual, such as susceptible-infectedsusceptible (SIS), susceptible-infected-recovered (SIR), susceptible-infected-removed-susceptible (SIRS), etc. The theoretical analysis of the behavior of these compartmental models in complex networks starts from the application of the heterogeneous mean-field (HMF) theory [12]. This formalism is based on the assumptions that all vertices with the same number of connections (i.e. within the same degree class) share the same dynamical properties, and that fluctuations are not important, and therefore all rel- 
evant variables can be described in terms of deterministic rate equations. The first assumption becomes natural once we admit that the degree is the only parameter describing the state of a vertex. On the other hand, the second assumption finds support in the small-world property shown by most complex networks [23, implying that dynamical fluctuations take place so close together that they can be washed away in very few time step 2 . HMF has proved to be extremely useful in providing an accurate description of epidemic models on complex networks, and has in fact become the de facto standard tool to analyze general non-equilibrium processes on such substrates [1].

In this paper we will pay attention on a non-equilibrium dynamical model with relevance both in epidemic modeling and other ambits of non-equilibrium statistical physics, namely the forest fire model (FFM). First introduced in 1992 by Bak et al. [25, and further developed by Drossel and Schwabl 26, the FFM was elaborated to show selforganized criticality and avalanche behavior in a specific limit of its defining parameters. Even though its general status as a self-organized critical model is under debate [27/28, it has found successful applications as a general disease propagation model [12]29, in which susceptible individuals can get the disease either by transmission from an infected neighbor or spontaneously (because of a mosquit bite for instance), while recovered individuals can become again susceptible. It is thus akin to a SIRS model [12] with an external source of infected individuals. More interestingly, it encompasses several other compartmental models, which can be recovered in a convenient way as certain limits of the parameters that define the FFM.

Previous works on the FFM in complex networks have reported, among other results, the presence of self-sustained oscillations in small-world networks [30] and analyzed the distribution of excitations depending on topology [31. From the perspective of the SIRS model, on the other hand, its epidemiological implications have been discussed for certain ranges of its parameter space 3233. In the present paper we provide an extensive theoretical analysis of the FFM, using the HMF formalism and focusing on the steadystate dynamics of the model in the whole range of its parameter space. Our analysis allows to emphasize its interpretation, in the different regimes, in terms of known epidemic models, providing analytic expressions for the steady-state density of infected individuals in certain limits of the relevant parameters. A comparison of the theoretical results with extensive numerical simulations, allows finally to unveil the limitations of the HMF approach in this and probably other epidemic models, hinting towards the break down of HMF theory when dynamical correlations become relevant 24 .

We organized our paper as follows: In Sec. 2 we define the FFM, discussing its relation to self-organized criticality and disease propagation. In Sec. [3 we develop the HMF theory of the FFM in general complex networks. Sec. [4 deals with the steady-state solution of the HFM equations obtained before. We show in particular how an ap-

\footnotetext{
${ }^{2}$ As a matter of fact, fluctuations can be shown to be irrelevant in some particular cases [24].
}

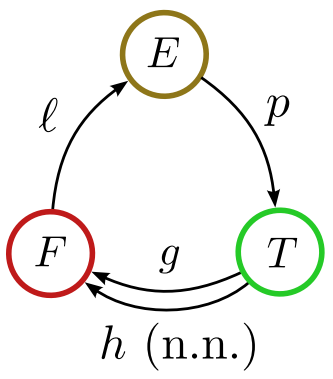

Fig. 1. Representation of the three states present in the forest fire model, and of its dynamical rules. (n.n. stands for a "nearest neighbor" interaction).

propriate rescaling of the equations allows to simplify the description and to conveniently reduce the number of degrees of freedom. A general analysis is presented for both homogeneous networks and heterogeneous networks with no spontaneous infection. An explicit analysis of uncorrelated SF networks is presented in Sec. 5. The numerical simulations shown in Sec. [6 allow us to check the validity of our theoretical results, as well as to draw the limits of validity of general HMF approaches. Finally, we present our conclusions in Sec. 7

\section{Forest fire model on complex networks}

We consider the FFM on general complex networks which, from a statistical point of view, are described at a coarsegrained level by the degree distribution $P(k)$ and the degreedegree correlations, given by the conditional probability $P\left(k^{\prime} \mid k\right)$ that a vertex of degree $k$ is connected to a vertex of degree $k^{\prime}$ 34.

In the FFM, each vertex in the network is in one of three excluding states: E (empty), T (tree), F (burning tree). The evolution of the model is defined in a continuous time formulation in terms of the possible events that can happen in a small time interval $\Delta t$ (see Fig. 1)

1. $\mathrm{E} \rightarrow \mathrm{T}: \mathrm{A}$ tree can grow on an empty vertex with probability $p \Delta t$.

2. $\mathrm{T} \rightarrow \mathrm{F}$ : For a tree, each of its burning neighbors (if any) can light it with probability $h \Delta t$.

3. $\mathrm{T} \rightarrow \mathrm{F}:$ Additionally, there is also a probability $g \Delta t$ for a tree to catch fire spontaneously (e.g. mediating a lightning). These two burning events are considered probabilistically independent.

4. $\mathrm{F} \rightarrow \mathrm{E}:$ A burning tree leaves an empty vertex with probability $\ell \Delta t$.

The FFM was proposed to exhibit self-organized criticality in the double limit $p \rightarrow 0$ and $g / p \rightarrow 0$, in which clusters of trees are allowed to grow before burning down, leading to a distribution of fire avalanches with an approximately power-law form [35]. For finite $p$ and $g$ values, an activated dynamics with no evidence of avalanches is instead observed 3031. 
From the point of view of disease modeling, trees, fires, and empty sites represent, respectively, susceptible, infected, and recovered individuals. Within this interpretation of the model, susceptible individuals can acquire the disease by contact with one or more infected individuals at rate $h$; infected individuals recover in a time scale of the order $\sim 1 / \ell$; recovered individuals become again susceptible in a time scale $\sim 1 / p$; and healthy individuals become spontaneously infected in a time scale $\sim 1 / \mathrm{g}$. With a set of 4 parameters, a unified description of the most usual epidemic models is achieved. Thus, in the limit $p=g=0$, we recover the SIR model; the limit $g=0$ and $p \rightarrow \infty$ leads to the SIS model; while the limit $g=0$ corresponds to the SIRS model.

Note finally that our definition of the FFM considers time as a continuous variable, in opposition to previous approaches. This formulation is preferred in order to lead more naturally to a continuous analytical description in terms of differential equations, and will be taken into account when performing numerical simulations in Sec. 6 .

\section{Heterogeneous Mean-Field theory for the FFM in complex networks}

Within the HMF approach, a dynamical system is assumed to be fully determined in terms of the relative probabilities that a vertex of given degree $k$ is in any one of the states allowed by the dynamics [1416]. In the case of the FFM, this description invokes the partial densities $\rho_{k}^{\alpha}(t)$, defined as the conditional probability that a vertex of degree $k$ is, at time $t$, in the state $\alpha$, with $\alpha \in\{E, T, F\}$. Since each vertex must be in one of these states, the partial densities satisfy the normalization condition

$$
\rho_{k}^{E}(t)+\rho_{k}^{T}(t)+\rho_{k}^{F}(t)=1 .
$$

Therefore, only two independent partial densities, say $\rho_{k}^{E}(t)$ and $\rho_{k}^{F}(t)$, must be considered in the analysis. On the other hand, the density of vertices in each state at time $t$ is given by

$$
\rho^{\alpha}(t)=\sum_{k} P(k) \rho_{k}^{\alpha}(t) .
$$

At the core of the HMF theory lie the rate equations fulfilled by the partial densities. By considering the different microscopic steps allowed in the model we can readily write the change of the quantities $\rho_{k}^{\alpha}(t)$ in an infinitesimal time step $\Delta t$, that are given by

$$
\begin{aligned}
\rho_{k}^{E}(t+\Delta t) & =\rho_{k}^{E}(t)+\rho_{k}^{F}(t) \operatorname{Prob}_{k}(F \rightarrow E) \\
& -\rho_{k}^{E}(t) \operatorname{Prob}_{k}(E \rightarrow T), \\
\rho_{k}^{T}(t+\Delta t) & =\rho_{k}^{T}(t)+\rho_{k}^{E}(t) \operatorname{Prob}_{k}(E \rightarrow T) \\
& -\rho_{k}^{T}(t) \operatorname{Prob}_{k}(T \rightarrow F), \\
\rho_{k}^{F}(t+\Delta t) & =\rho_{k}^{F}(t)-\rho_{k}^{F}(t) \operatorname{Prob}_{k}(E \rightarrow F) \\
& +\rho_{k}^{E}(t) \operatorname{Prob}_{k}(T \rightarrow F),
\end{aligned}
$$

where $\operatorname{Prob}_{k}(\alpha \rightarrow \beta)$ is the probability that a vertex of degree $k$ experiences the transition from the state $\alpha$ to the state $\beta$ in a time interval $\Delta t$. From the definition of the FFM in Sec. 2, we can immediately write down $\operatorname{Prob}_{k}(E \rightarrow T)=p \Delta t$ and $\operatorname{Prob}_{k}(F \rightarrow E)=\ell \Delta t$. In order to construct the term $\operatorname{Prob}_{k}(T \rightarrow F)$, we must consider that rules 2 and 3 defining the model, which represent a tree catching fire, are statistically independent. Therefore if we define $H$ as the event "A tree is lighted by its neighbors" and $G$ as the event "A tree lights up spontaneously", we have that $\operatorname{Prob}_{k}(T \rightarrow F)=$ $\operatorname{Prob}_{k}(G \cup H)=\operatorname{Prob}_{k}(H)\left[1-\operatorname{Prob}_{k}(G)\right]+\operatorname{Prob}_{k}(G)$. Rule 3 gives $\operatorname{Prob}_{k}(G)=g \Delta t$. Now, since in this description vertices with the same degree are statistically equivalent, the state of a given vertex is independent on the state of its neighbors, and it only depends on its degree. This allows us to write the probability for one neighbor of a tree with degree $k$ to be burning as

$$
\theta_{k}=\sum_{k^{\prime}} P\left(k^{\prime} \mid k\right) \rho_{k^{\prime}}^{F}
$$

given in terms of the average of the conditional probability $P\left(k^{\prime} \mid k\right)$ that the vertex $k$ is connected to a vertex of degree $k^{\prime}$, times the probability that this last vertex is burning, $\rho_{k^{\prime}}^{F}$. Notice that here we are assuming that the edge through which $k^{\prime}$ became burning is immediately available to transmit again the fire. This assumption, in opposition to the behavior of the SIR model [36], will thus be valid only for $p>0$.

From rule 2, the probability that a particular nearest neighbor fire ignites a tree in a vertex of degree $k$ is given by $h \Delta t \theta_{k}$. Therefore, the probability that a tree of degree $k$ is ignited by any of its nearest neighbors is $\operatorname{Prob}_{k}(H)=$ $1-\left[1-h \Delta t \theta_{k}\right]^{k}$. Substituting this expressions in Eqs. (3)(5), and taking the limit $\Delta t \rightarrow 0$, we obtain the final HMF equations for the FFM,

$$
\left\{\begin{array}{l}
\dot{\rho}_{k}^{E}(t)=\rho_{k}^{F}(t)-p \rho_{k}^{E}(t) \\
\dot{\rho}_{k}^{T}(t)=p \rho_{k}^{E}(t)-\rho_{k}^{T}(t)\left[h k \sum_{k^{\prime}} P\left(k^{\prime} \mid k\right) \rho_{k^{\prime}}^{F}(t)+g\right] \\
\dot{\rho}_{k}^{F}(t)=\rho_{k}^{T}(t)\left[h k \sum_{k^{\prime}} P\left(k^{\prime} \mid k\right) \rho_{k^{\prime}}^{F}(t)+g\right]-\rho_{k}^{F}(t)
\end{array}\right.
$$

where we have set $\ell=1$, which amounts to a trivial rescaling of time.

Eqs. (77) represent a complete description of the FFM at the HMF level. Even though we have derived them in a phenomenological way [1437, they can also be obtained from a microscopic point of view, considering explicitly the state of each vertex evolving as a Poisson random process 24,38. The mean-field result is then recovered by averaging over the random processes and over the vertices with same degree.

One final warning comment is in order here, concerning the fact that, in writing Eqs. (7), we have neglected altogether dynamical correlations between adjacent vertices, assuming explicitly that the state of a vertex is independent of the state of its nearest neighbors. As we will see, this assumption is not correct, especially for low fire (infection) densities, when the positions of different fires are in fact strongly correlated, leading thus to a breakdown of the HMF theory predictions (see Sec. 6). 


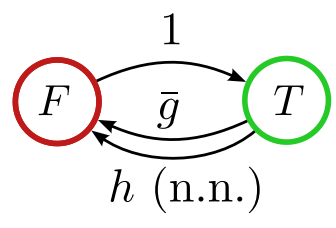

Fig. 2. Representation of the two states present in the SIS $+g$ model, and of its dynamical rules. Its steady-state is directly related to that of the FFM. (n.n. stands for a "nearest neighbor" interaction).

\section{Steady-state solution in general networks}

Let us consider the long time properties of the FFM. It typically corresponds to the steady-state calculated by setting $\dot{\rho}_{k}^{E}=\dot{\rho}_{k}^{T}=\dot{\rho}_{k}^{F}=0 \forall k$ in the HMF Eqs. (7), which yields the algebraic equations

$$
\left\{\begin{array}{l}
0=\rho_{k}^{F}-p \rho_{k}^{E} \\
0=p \rho_{k}^{E}-\rho_{k}^{T}\left[h k \sum_{k^{\prime}} P\left(k^{\prime} \mid k\right) \rho_{k^{\prime}}^{F}+g\right] \\
0=\rho_{k}^{T}\left[h k \sum_{k^{\prime}} P\left(k^{\prime} \mid k\right) \rho_{k^{\prime}}^{F}+g\right]-\rho_{k}^{F}
\end{array}\right.
$$

for the (now time-independent) variables $\rho_{k}^{\alpha}$. We look for nontrivial steady-states, so we will be concerned in searching solutions with $\rho_{k}^{\alpha} \neq 0$.

The analysis of Eqs. (8) can be simplified by noticing that the empty state plays the role of a rest state (c.f. Appendix A), which the system enters and leaves with constant rates. It can thus be factorized by writing its population density, from the first equation in (8), as $\rho_{k}^{E}=\rho_{k}^{F} / p$ and substituting it in the other two equations. Therefore, introducing the rescaling factor

$$
\eta=1+\frac{1}{p}
$$

and defining the new variable and parameter

$$
\bar{\rho}_{k}^{F} \equiv \eta \rho_{k}^{F}, \quad \bar{g} \equiv \eta g,
$$

we can consider the simplified set of equations

$$
\left\{\begin{array}{l}
0=\bar{\rho}_{k}^{F}-\rho_{k}^{T}\left[h k \sum_{k^{\prime}} P\left(k^{\prime} \mid k\right) \bar{\rho}_{k^{\prime}}^{F}+\bar{g}\right] \\
1=\bar{\rho}_{k}^{F}+\rho_{k}^{T}
\end{array}\right.
$$

as characterizing the steady-state of the FFM in a general complex network with a correlation pattern given by the conditional probability $P\left(k^{\prime} \mid k\right)$.

From Eq. (11), we can see that the steady-state of the FFM can be in general mapped to the steady-state of an SIS model [14 with a random source of infected individuals, arising from isolated susceptibles with rate $\bar{g}$. We can thus refer to it as a SIS + g model, see Fig. 2 In particular, setting $g=0$, the FFM becomes the SIRS model, which is therefore exactly mappable to the SIS model 32 .

In order to solve the set of Eqs. (11), one can proceed to substitute its second equation into its first one, to obtain $\bar{\rho}_{k}^{F}$ as a function of $\bar{\theta}_{k} \equiv \eta \theta_{k}$, namely

$$
\bar{\rho}_{k}^{F}=\frac{h k \bar{\theta}_{k}+\bar{g}}{1+\left(h k \bar{\theta}_{k}+\bar{g}\right)} .
$$

The equation is closed by expressing $\bar{\theta}_{k}$ self-consistently as

$$
\bar{\theta}_{k}=\sum_{k^{\prime}} P\left(k^{\prime} \mid k\right) \frac{h k^{\prime} \bar{\theta}_{k^{\prime}}+\bar{g}}{1+\left(h k^{\prime} \bar{\theta}_{k^{\prime}}+\bar{g}\right)} .
$$

Solving this equation for $\bar{\theta}_{k}$ directly gives the steady-state fire density, and thus the stationary solution of the process.

Prior to solving these equations, however, notice that Eqs. (11) imply that the solution for $\bar{\rho}_{k}^{F}$ takes the functional form $\bar{\rho}_{k}^{F}(\bar{g}, h)$, and is now independent of $p$ (namely of $\eta$ ). Therefore, we can write down a scaling solution for the steady-state fire density in the FFM in any network as

$$
\rho^{F}(\eta, g, h)=\frac{1}{\eta} \bar{\rho}^{F}(\eta g, h) .
$$

This scaling solution implies that the factor $p$ (growth of new trees) affects the model only by a rescaling of the fire density and of the rate of spontaneous lightning. This justifies the fact that no more than 2 out of the 4 initial parameters are relevant to the study of the stationary state of the FFM within the HMF theory approximation. For the particular case $g=0$, Eq. (14) leads to

$$
\rho^{F}(\eta, g=0, h) \equiv \frac{1}{\eta} F(h),
$$

that is, the fire density is a function of $h$, divided by $\eta$. In the limit $p \rightarrow \infty(\eta \rightarrow 1)$, we recover the standard SIS model. On the other hand, any finite $p$ will lead to a smaller value of the fire density. The fact that an upper bound on $\rho^{F}$ can be deduced from $\eta$ is in general valid for any $g$ : since $\bar{\rho}_{k}^{F} \leq 1$, given that it is a probability, we have that

$$
\rho^{F} \leq \frac{1}{\eta}=\frac{p}{p+1}
$$

This upper bound on $\rho^{F}$ has important consequences from a numerical point of view. In fact, even in the regions of the parameter space $(h, g)$ where a nonzero value of $\rho^{F}$ is expected, a very small value of $p$ will lead to a correspondingly small fire density, which can be difficult to measure unless in the limit of very large network sizes.

The factorization of the empty state, the functional form of the fire density and its upper bound in terms of $\eta$ are in fact quite general features, that can be found in any dynamical system having rest states, see Appendix A.

\subsection{Homogeneous networks}

Let us consider first the simplest situation of the FFM taking place in a homogeneous network, in which the degree distribution is peaked at an average degree $\langle k\rangle$ and decays exponentially fast for $k \ll\langle k\rangle$ and $k \gg\langle k\rangle$. We can approximate all vertices as having the same degree $k=\langle k\rangle$. In this case, we have $\rho_{k}^{F} \equiv \rho^{F}$ effectively independent of $k$, and also $\theta_{k}=\rho^{F}$. Eq. (12) thus takes the form

$$
h\langle k\rangle\left(\bar{\rho}^{F}\right)^{2}+(1+\bar{g}-h\langle k\rangle) \bar{\rho}^{F}-\bar{g}=0,
$$


whose only positive solution is

$$
\bar{\rho}^{F}=\frac{h\langle k\rangle-1-\bar{g}+\sqrt{-4 h\langle k\rangle+(1+\bar{g}+h\langle k\rangle)^{2}}}{2 h\langle k\rangle},
$$

as already shown in Ref. 39.

For $g>0$ the fire density is strictly positive. This results from the fact that the trees in the network can always ignite themselves with some nonzero probability, therefore always reviving the fire density. On the other hand, for $g=0$, the fire density takes the form

$$
\rho^{F}=\frac{|1-h\langle k\rangle|-(1-h\langle k\rangle)}{2 \eta h\langle k\rangle} .
$$

which is equal to 0 for $h\left\langle\langle k\rangle^{-1}\right.$ and positive otherwise. That is, the FFM experiences an absorbing-state phase transition [40] at a critical value $h_{c}=\langle k\rangle^{-1}$. For $h>h_{c}$, the systems is in an active phase, in which the fire activity never stops, taking the asymptotic form

$$
\rho^{F} \sim \frac{h-h_{c}}{\eta}
$$

On the other hand, for $h<h_{c}$, the system reaches an absorbing state, in which fire always ends up disappearing by lack of transmissibility.

\subsection{Phase transition for $g=0$ on complex networks}

For networks with a general degree distribution $P(k)$ and general correlation pattern $P\left(k^{\prime} \mid k\right)$, the explicit solution of Eqs. (12) and (13) becomes a quite difficult task. It is possible, however, to obtain information for a general network in the particular case $g=0$. Setting $g$ to 0 changes the forest fire model to a SIRS model, whose stationary state can be related to the one of the SIS model by removal of the rest state, see Eq. (11). Therefore, in this particular limit, the FFM exhibits an absorbing-state phase transition between an active (burning, infected) phase and an absorbing (fire-free, healthy) phase, located at the critical point 41

$$
h_{c}=\frac{1}{\Lambda_{m}},
$$

where $\Lambda_{m}$ is the largest eigenvalue of the connectivity matrix $C_{k k^{\prime}}=k P\left(k^{\prime} \mid k\right)$. Interestingly, this threshold is independent of the rate $p$ of creation of new trees, which only affects the overall density of fires, as expressed in Eq. (15).

\section{Explicit solution for uncorrelated scale-free networks}

In order to obtain explicit analytical results for the HMF equations of the FFM, we restrict ourselves to the case of uncorrelated networks. In this case, the conditional probability $P\left(k^{\prime} \mid k\right)$ takes the form $P\left(k^{\prime} \mid k\right)=k^{\prime} P\left(k^{\prime}\right) /\langle k\rangle$ [4. $\bar{\theta}_{k}$ thus becomes independent of $k$ :

$$
\bar{\theta}_{k} \equiv \bar{\theta}=\frac{1}{\langle k\rangle} \sum_{k} k P(k) \bar{\rho}_{k}^{F} .
$$

From Eq. (12), $\bar{\rho}_{k}^{F}$ is now an algebraic function of $k$. For the interesting case of SF networks, with a degree distribution $P(k)=(\gamma+1) m^{\gamma+1} k^{-2-\gamma}$ in the continuous degree approximation, where $m$ is the minimum degree present in the network, Eq. (13) reads, replacing summations by integrals,

$$
\begin{aligned}
\bar{\theta}= & \gamma m^{\gamma} \int_{m}^{\infty} \frac{h \bar{\theta} k^{-\gamma}+\bar{g} k^{-1-\gamma}}{1+\eta(h k \bar{\theta}+\bar{g})} \\
= & F\left(1, \gamma ; \gamma+1 ;-\frac{1+\bar{g}}{m h \bar{\theta}}\right) \\
& +\frac{\gamma \bar{g}}{(\gamma+1) m h \bar{\theta}} F\left(1, \gamma+1 ; \gamma+2 ;-\frac{1+\bar{g}}{m h \bar{\theta}}\right)
\end{aligned}
$$

where $F(a, b ; c ; z)$ is the Gauss hypergeometric function 42 . Using the power series development of the hypergeometric function [42] or the asymptotic expression

$$
F\left(1, \gamma ; 1+\gamma ;-z^{-1}\right)=\gamma \sum_{n=1}^{\infty}(-1)^{n} \frac{z^{n}}{n-\gamma}+\frac{\gamma \pi}{\sin (\gamma \pi)} z^{\gamma},
$$

valid for $\arg (z)<\pi$ and $\gamma \notin \mathbb{N}$, one finds the self-consistent equation for $\bar{\theta}$ to be:

$$
\bar{\theta}=\frac{\bar{g}}{1+\bar{g}}+\frac{1}{1+\bar{g}} F\left(1, \gamma ; \gamma+1 ;-\frac{1+\bar{g}}{m h \bar{\theta}}\right),
$$

for all $\frac{1+\bar{g}}{m h \theta}>0$. This is the final equation we need to solve in order to find the steady state fire density. Note that the condition of validity $\gamma \notin \mathbb{N}$ is no more a restriction here, by analytical continuation of the hypergeometric function.

Before proceeding, let us express more explicitly the dependence of the fire density (what we are ultimately interested in) and the probability for a neighbor to burn, $\bar{\theta}$. We can directly calculate it using Eq. (12) and a similar reasoning as before, to obtain:

$$
\begin{aligned}
\bar{\rho}^{F} & =\sum_{k} P(k) \bar{\rho}_{k}^{F}=(\gamma+1) m^{\gamma+1} \int_{m}^{\infty} \frac{h k \bar{\theta}+\bar{g}}{1+\eta(h k \bar{\theta}+\bar{g})} \\
& =\frac{\bar{g}}{1+\bar{g}}+\frac{1}{1+\bar{g}} F\left(1, \gamma+1 ; \gamma+2 ;-\frac{1+\bar{g}}{m h \bar{\theta}}\right) .
\end{aligned}
$$

Now, using one of Gauss's relations for contiguous hypergeometric functions [42], namely

$(a-c) z F(a, b ; c+1 ; z)+c F(a, b-1 ; c ; z)=c(1-z) F(a, b ; c ; z)$

with $a=1, b=\gamma+1$ and $c=\gamma+1$, the hypergeometric function can be re-expressed directly in terms of $\bar{\theta}$ as obtained in Eq. (26) to get

$$
\bar{\rho}^{F}=\frac{\bar{g}}{1+\bar{g}}+\frac{\gamma+1}{\gamma} \frac{m h \bar{\theta}}{1+\bar{g}}(1-\bar{\theta})
$$

In a general SF network without correlations, the fire density is thus a quadratic function of the probability $\bar{\theta}$. 


\subsection{Exact solution for $\gamma=1$}

In the case $\gamma=1$ it is possible to solve exactly the selfconsistent equation Eq. (26), which takes the form 42

$$
\bar{\theta}=\frac{\bar{g}}{1+\bar{g}}+\frac{m h \bar{\theta}}{(1+\bar{g})^{2}} \ln \left(1+\frac{1+\bar{g}}{m h \bar{\theta}}\right) .
$$

Introducing the new variable $y=\bar{g}+\frac{\bar{g}(1+\bar{g})}{m h \theta}$ the equation becomes

$$
y e^{y}=\bar{g} e^{\bar{g}+\frac{(1+\bar{g})^{2}}{m h}} .
$$

Recognizing the solution of $y e^{y}=x$ as the W Lambert function, $y=W(x)$ [43, the result follows:

$$
\bar{\theta}=\frac{1+\bar{g}}{m h}\left[-1+\bar{g}^{-1} W\left(\bar{g} e^{\bar{g}+\frac{(1+\bar{g})^{2}}{m h}}\right)\right]^{-1}
$$

The value of the fire density is then obtained by plugging this expression into Eq. (29). Expanding the fire density at first order for small $\bar{g}$ yields then

$$
\begin{aligned}
\bar{\rho}^{F} & \simeq \frac{2 h m\left(e^{\frac{1}{h m}}-1\right)-2}{h m\left(e^{\frac{1}{h m}}-1\right)^{2}}+\left[3+\frac{8}{h^{2} m^{2}\left(e^{\frac{1}{h m}}-1\right)^{3}}\right. \\
& \left.+\frac{8-10 h m}{h^{2} m^{2}\left(e^{\frac{1}{h m}}-1\right)^{2}}-\frac{8-2 h m}{h m\left(e^{\frac{1}{h m}}-1\right)}\right] \bar{g},
\end{aligned}
$$

where the first term corresponds, obviously, to the SIS result 14, recovered in the limit $g \rightarrow 0$. The expansion in terms of $h$ is

$$
\bar{\rho}^{F} \simeq \frac{\bar{g}}{1+\bar{g}}+\frac{2 \bar{g} m}{(1+\bar{g})^{3}} h,
$$

yielding a nonzero fire density (an infected steady-state) for any value of $h$ if $\bar{g}>0$.

\subsection{Asymptotic solution for $\gamma \neq 1$}

Let us now turn our attention to the behavior of the FFM in the general case $\gamma \neq 1$. To do so, we will study the limit of low fire density, namely $\bar{\theta} \ll 1$ and $\bar{g} \ll 1$. In this limit, Eq. (29) leads to

$$
\bar{\rho}^{F} \sim \bar{g}+\frac{\gamma+1}{\gamma} m h \bar{\theta},
$$

so we need to develop the self-consistent equation for $\bar{\theta}$, Eq. (26), up to the first most relevant terms, using the asymptotic expansion Eq. (25). For $\bar{g}=0$ we recover the known result $\bar{\rho}^{F} \sim\left(h-h_{c}\right)^{\beta}$ [37, where

$$
h_{c}=\left\{\begin{array}{lll}
0 & \text { for } & 0<\gamma<1 \\
\frac{\gamma-1}{\gamma m} & \text { for } & \gamma>1
\end{array},\right.
$$

and

$$
\beta=\left\{\begin{array}{lll}
\frac{1}{1-\gamma} & \text { for } & 0<\gamma<1 \\
\frac{1}{\gamma-1} & \text { for } & 1<\gamma<2 \\
1 & \text { for } & \gamma>2
\end{array}\right.
$$

For $\bar{g}>0$, let us consider separately each possible value of $\gamma$.

(1) $0<\gamma<1$ :

In this case, the leading approximation for $\bar{\theta}$ is

$$
\bar{\theta} \simeq \frac{\bar{g}}{1+\bar{g}}+\frac{\gamma \pi}{(1+\bar{g}) \sin (\gamma \pi)}\left(\frac{m h \bar{\theta}}{1+\bar{g}}\right)^{\gamma}
$$

which is valid for $\frac{m h \bar{\theta}}{1+\bar{g}} \ll 1$. If $\bar{g} \neq 0$, the solution of $\bar{\theta}$ depends in a nontrivial way on both $\bar{g}$ and $h$ as

$$
\bar{\theta}=\kappa\left(\frac{\bar{g}}{1+\bar{g}}, \frac{\gamma \pi}{(1+\bar{g}) \sin (\gamma \pi)}\left[\frac{m h}{1+\bar{g}}\right]^{\gamma}\right)
$$

where the function $\kappa(a, b)$ is defined as the solution of the implicit equation $\kappa(a, b)=a+b \cdot \kappa(a, b)^{\gamma}$. In Appendix B we give an explicit expression for $\kappa(a, b)$ in terms of $a$ and $b$. For small $\bar{g}$ and $h$, two different regimes can be isolated from the development of the $\kappa$ function (71):

$-h \ll \bar{g}^{\frac{1-\gamma}{\gamma}}$ : The most significant terms are now :

$$
\bar{\theta} \simeq \frac{\bar{g}}{1+\bar{g}}+\frac{\gamma \pi}{(1+\bar{g}) \sin (\gamma \pi)}\left(\frac{m h \bar{g}}{(1+\bar{g})^{2}}\right)^{\gamma},
$$

so that, to leading order in $h$, the fire density goes like:

$$
\bar{\rho}^{F} \simeq \frac{\bar{g}}{1+\bar{g}}+\frac{\gamma+1}{\gamma} \frac{m \bar{g}}{(1+\bar{g})^{3}} h .
$$

The introduction of an infinitesimal $\bar{g}>0$ destroys again the absorbing-state phase transition, since both terms in Eq. (41) are positive.

$-\bar{g} \ll h^{\frac{\gamma}{1-\gamma}}$ : In this case, the leading terms are:

$$
\bar{\theta} \simeq \bar{\theta}_{0}+(1-\gamma)^{-1} \bar{g}
$$

where $\bar{\theta}_{0}=\left(\frac{\gamma \pi(m h)^{\gamma}}{\sin (\gamma \pi)}\right)^{\frac{1}{1-\gamma}}$. Thus, Eq. (29) yields

$\bar{\rho}^{F} \simeq \frac{\gamma+1}{\gamma} m h \theta^{0}\left(1-\bar{\theta}_{0}\right)+\left[1+\frac{(1+\gamma) m h}{\gamma(1-\gamma)}\left(1-2 \theta_{0}\right)\right] \bar{g}$.

One should notice that in order to derive this last expression we used Eq. (38), which assumes $\frac{m h \bar{\theta}}{1+\bar{g}} \ll 1$, so not only do we need $\bar{g}$ to be small in order for this approximation to hold, but $h$ cannot be too large either.

(2) $\gamma>1$ :

The self-consistent equation Eq. (26) can be approximated in this regime by:

$$
\bar{\theta} \simeq \frac{\bar{g}}{1+\bar{g}}+\frac{\gamma}{\gamma-1} \frac{m h \bar{\theta}}{(1+\bar{g})^{2}} .
$$


So we obtain:

$$
\bar{\theta} \simeq \frac{(\gamma-1) \bar{g}(1+\bar{g})}{(\gamma-1)(1+\bar{g})^{2}-\gamma m h}
$$

and to first order, the fire density is given by

$$
\bar{\rho}^{F} \simeq \bar{g}(1-\bar{g}+2 m h) .
$$

To summarize, in all cases considered above the presence of a fire lightning probability $g>0$ eradicates any phase transition in the model, just like on homogeneous networks, Sec. 4.1 rendering a nonzero steady-state that grows, at lowest order, linearly with $\bar{g}$, with a numerical prefactor which is a complex function of $h$, depending on the particular value of $\gamma$ considered.

\section{Numerical Simulations}

In order to check the analytic predictions developed in the previous sections, we performed extensive numerical simulations of the FFM on top of different network models, both homogeneous and heterogeneous. Simulations were implemented using a sequential update algorithm [40. Given a substrate network, each vertex is first randomly initialized in one of the three possible states, empty, tree, or fire. In the dynamics, every time step a vertex is chosen at random and its state is updated applying the rules defined in Sec. 2 with given reaction rates $\ell, p, g$ and $h$. In principle, to reproduce the exact dynamics a time step would correspond to a time increment $\Delta t=1 / N$ [40]. To speed up the arrival to the stationary state, we chose $\Delta t$ larger, but still small enough so as to keep the process random. Typically, the probabilities involved in the evolution are such that $\max (\ell \Delta t, p \Delta t, g \Delta t, h \Delta t) \leq 0.1$. We have checked that smaller probabilities do not affect the properties of the steady state, but only slow down the transient to reach it. We can recover the correct theoretical expressions from our HMF analysis just by substituting the model parameters by the rescaled values $h / \ell, g / \ell$, $p / \ell$, and, correspondingly, $\eta=1+\ell / p$.

\subsection{Homogeneous networks: The Watts-Strogatz model}

As an example of a homogeneous network, we consider the small-world model proposed by Watts and Strogatz (WS) [23]. Networks in this model are generated as follows: The starting point is a ring with $N$ vertices, in which every vertex is symmetrically connected to its $2 K$ nearest neighbors. Then, for every vertex, each edge connected to a clockwise neighbor is rewired to a randomly chosen vertex with probability $p_{r w}$, and kept with probability $1-p_{r w}$. This procedure generates a graph with a degree distribution that decays faster than exponentially for large $\mathrm{k}$, and average degree $\langle k\rangle=2 K$. We considered here WS networks with $p_{r w}=1$ and $K=3$.

In Fig. 3 we show the numerical results obtained in WS homogeneous networks of size $N=10^{5}$, with fixed
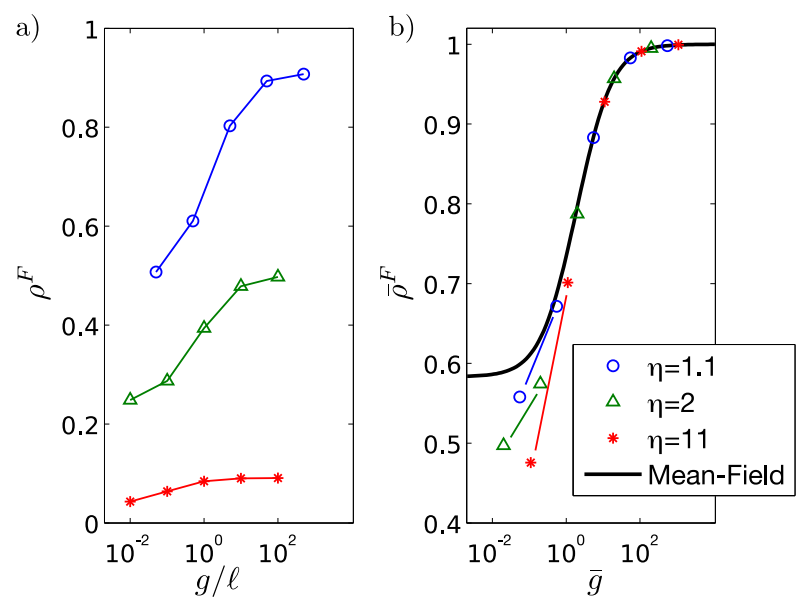

Fig. 3. Steady state fire density in the FFM on homogeneous WS networks of size $N=10^{5}$. (a) Raw data for different values of $\eta$ as a function of $g / \ell$, and fixed $h / \ell=0.4$. (b) Data collapse of the previous plots as given by Eq. (47). The functions agree very well with the prediction by HFM theory for large values of the ratio $\bar{g} \equiv \eta g / \ell$. The separation of the curves at low $\bar{g}$ indicates a possible non-mean-field regime.

parameters $\ell=10^{-3}$ and $h / \ell=0.4$, and varying values of $p$ and $g$. We chose in particular a large value of $h / \ell$, larger than $h_{c} / \ell=\langle k\rangle^{-1}=1 / 6$, in order to avoid possible problems in the vicinity of the critical point for small values of $g$. In this case, the theoretical prediction for the fire density is given by Eq. (18), with the correct rescaling of parameters

$$
\rho^{F}=\frac{\frac{6 h}{\ell}-1-\eta \frac{g}{\ell}+\sqrt{-24 \frac{h}{\ell}+\left(1+\eta \frac{g}{\ell}+6 \frac{h}{\ell}\right)^{2}}}{12 \frac{h}{\ell} \eta},
$$

indicating, as argued in Sec. 4, that $\eta \rho^{F}$ should be a scaling function of $\eta g / \ell$. As we can observe in Fig. 3(b), the theoretical prediction is very well satisfied by numerical data for large values of $\eta g / \ell$, collapsing all plots on the functional form of Eq. (47) when the appropriate rescaling is performed. However, for values of $\eta g / \ell \leq 10$, we also observe a noticeable departure from the mean-field prediction, which is more conspicuous for large values of $\eta$. One could naively attribute this departure to a simple numerical artifact: since we have in general that $\rho^{F} \sim \eta^{-1}$, one could argue that, for fixed $h, g$, and $\ell$, larger values of $\eta$ lead to ever smaller fire densities. Therefore, for sufficiently large $\eta$, we could expect such small fire density that its steady state determination will incur in numerical resolution problems, unless extremely large network sizes are considered. But here the minimal fire density we find is of the order $\sim 0.04 \gg \frac{1}{N}=10^{-5}$ and so the number of burning trees still represents a significant fraction of the total population.

A more thorough analysis, however, reveals that the actual reason of this departure is the failure of the meanfield assumption of lack of dynamical correlations between vertices discussed in Sec. [3 in the limit of small $\eta g / \ell$, and for large $\eta$ (small $p / \ell$ ). In this case, the fire density is very 
a)
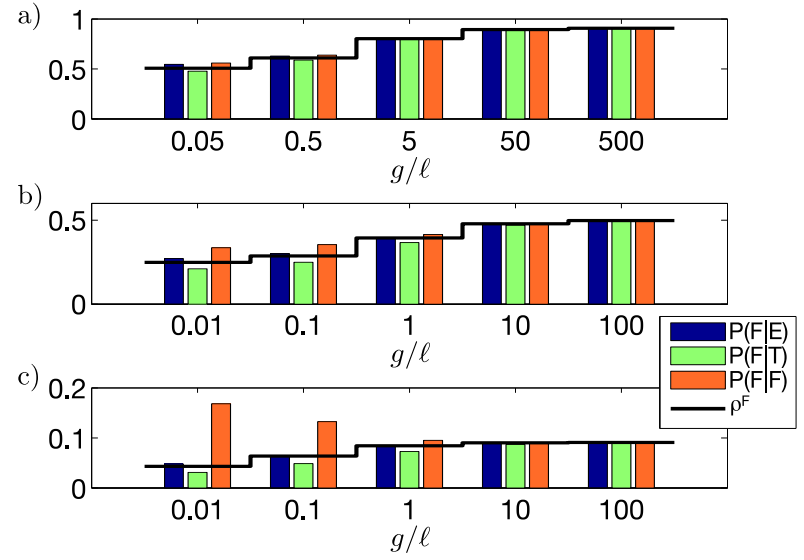

Fig. 4. Dependence of a vertex's state on his neighbors' states in WS networks of size $N=10^{5}$. The three graphs correspond to the three different curves presented in Fig 3 a) $\eta=1.1$, b) $\eta=2$, c) $\eta=11$. The red curve is the fire density $\rho^{F}$ in each case. In the absence of state correlations, we should have $P(F \mid E)=P(F \mid T)=P(F \mid F)=\rho^{F}$.

small, and therefore burning vertices are very likely nearest neighbors of other fires, precisely those that originated them. This fact introduces correlations between the state of nearest neighbors which invalidate the whole mean-field approximation. We can check this argument in a homogeneous network by comparing the probability $P(\alpha)$ that any vertex is in state $\alpha$, with the conditional probability $P(\alpha \mid \beta)$ that a vertex is in state $\alpha$, provided it is nearest neighbor of a vertex in state $\beta$. In Fig. 4 we compare the numerical values of $P(F) \equiv \rho^{F}$ with the corresponding conditional probabilities $P(F \mid E), P(F \mid T)$ and $P(F \mid F)$ in simulations performed for different values of $\eta$, plotted as a function of $g / \ell$. The figure shows that $P(F \mid F)$ is clearly larger that $\rho^{F}$ in the areas of dissension: for small $g / \ell$, and especially for the largest values of $\eta$. This results confirms the presence of strong dynamical correlations between vertices, and hints towards the failure of the HMF approximation in this region of the parameter space.

\subsection{Heterogeneous networks: The Barabási-Albert model}

The Barabási-Albert (BA) model is an algorithm to generate growing SF networks with degree exponent $\gamma=1$, based on the preferential attachment paradigm [3]. This model is defined as follows: we start from a small number $m_{0}$ of vertices, and at each time step, a new vertex is introduced, with $m$ edges that are connected to old vertices $i$ with probability $\Pi\left(k_{i}\right)=k_{i} / \sum_{j} k_{j}$, where $k_{i}$ is the degree of the $i^{\text {th }}$ vertex. After iterating this procedure a large number of times, we obtain a network composed by $N$ vertices, minimum degree $m$, fixed average degree $\langle k\rangle=2 m$, degree distribution $P(k)=2 m^{2} k^{-3}$ and almost vanishing degree correlations 4445]. The simulations considered here were perform with $m=2$ and a network size $N=10^{6}$.
In the case of the BA network, we can check the accuracy of the exact HMF solution for $\gamma=1$, as given by Eqs. (29) and (32). This is a nontrivial function of two variables, $g$ and $h$. Therefore, to check it in a simple way, we focused on the small $g$ and $h$ regimes, in which expressions (33) and (34) should provide a good approximation. In particular, we computed the quantities $\partial \bar{\rho}^{F} / \partial \bar{g}$ and $\partial \bar{\rho}^{F} / \partial h$ which, for small $h / \ell$ and $\bar{g}$ can be approximated by

$$
\begin{aligned}
\left.\frac{\partial \bar{\rho}^{F}}{\partial \bar{g}}\right|_{\bar{g}=0}= & 3+\frac{8}{h^{2} m^{2}\left(e^{\frac{1}{h m}}-1\right)^{3}}+\frac{8-10 h m}{h^{2} m^{2}\left(e^{\frac{1}{h m}}-1\right)^{2}} \\
& -\frac{8-2 h m}{h m\left(e^{\frac{1}{h m}}-1\right)}, \\
\left.\frac{\partial \bar{\rho}^{F}}{\partial h}\right|_{h=0}= & \frac{2 \bar{g} m}{(1+\bar{g})^{3}} .
\end{aligned}
$$

To obtain those quantities numerically, we used computer simulations to find the stationary fire density for several values of the variable with respect to which we took the partial derivative. Those values were chosen sufficiently small $\left(\bar{g}<10^{-3}\right.$ and $\left.h / \ell<2 \cdot 10^{-3}\right)$ so that the expected second order term is negligible. We then checked that the points formed the expected straight line, on which we measured the slope. The error on this slope comes from statistical uncertainties on the measured fire density for the points considered.

Figs. 5 and 6 show these quantities, computed from numerical simulations of the FFM in BA networks with $\ell=10^{-2}$. In order to ensure that we are within the region of validity of the HMF prediction, we chose a small value of $\eta=1.1(p / \ell=10)$. The good agreement observed between numerical simulations and the theoretical HMF predictions in Eqs. (48) confirms the validity of the HMF analysis in this parameter regime.

\subsection{Heterogeneous networks: The uncorrelated configuration model}

To generate SF networks with an arbitrary degree exponent $\gamma \neq 1$, we used the uncorrelated configuration model (UCM) [46]. This model is defined as follows: We start from $N$ initially disconnected vertices. Each vertex $i$ is assigned a degree $k_{i}$, extracted from the probability distribution $P(k) \sim k^{-2-\gamma}$, subject to the constraints $m \leq k_{i} \leq N^{1 / 2}$ and $\sum_{i} k_{i}$ even. Finally, the actual network is constructed by randomly connecting the vertices with $\sum_{i} k_{i} / 2$ edges, respecting the preassigned degrees and avoiding multiple and self-connections. Using this algorithm, it is possible to create SF networks whose average maximum degree (or cut-off) scales as $k_{c}(N) \sim N^{1 / 2}$ for any degree exponent $\gamma$, and which are completely uncorrelated [47. In the present simulations we chose a minimum degree $m=2, \ell=10^{-2}$ and sizes up to $N=10^{6}$. 


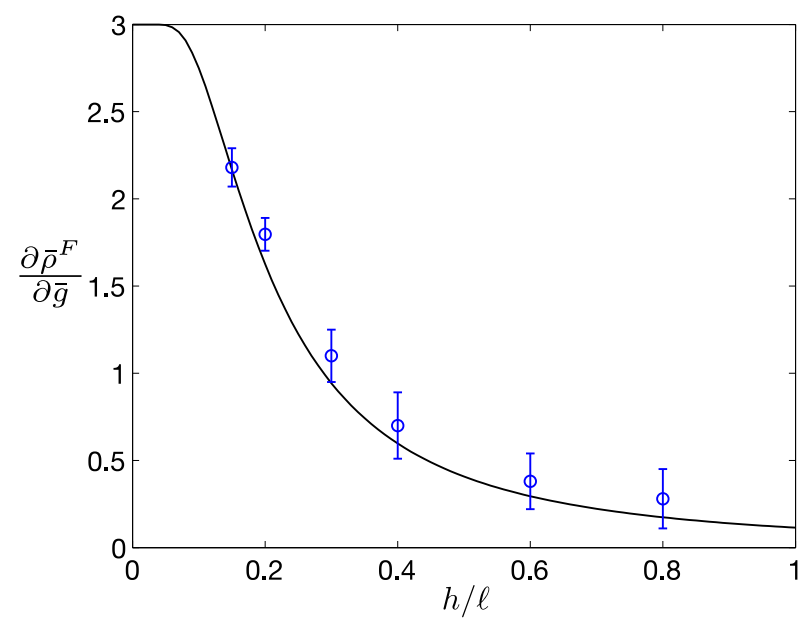

Fig. 5. Slope $\frac{d \bar{\rho}_{f}}{d \bar{g}}$ as a function of $h / \ell$ evaluated at $\bar{g} \leq 0.001$ on BA networks. The thick line is the mean field prediction for $\bar{g}=0$, Eq. (48).

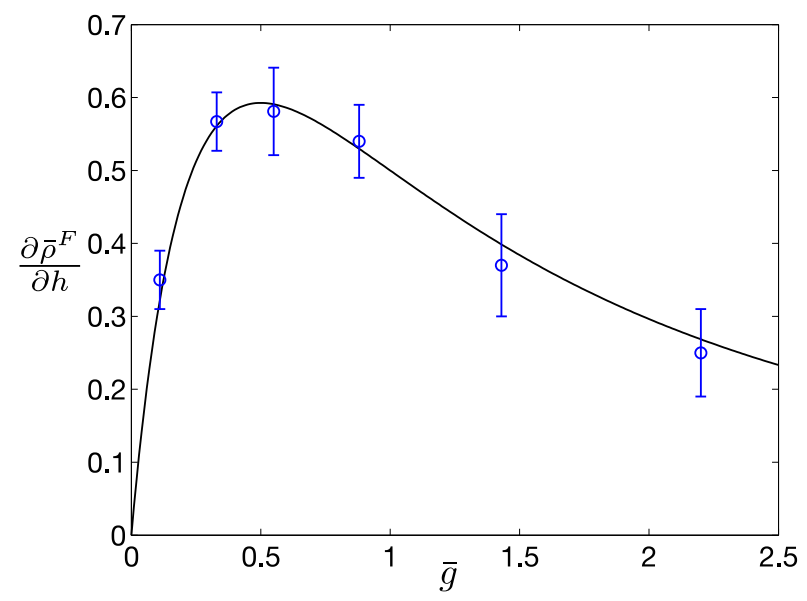

Fig. 6. Slope $\frac{d \bar{\rho}_{f}}{d h}$ as a function of $\bar{g}$ evaluated at $h / \ell \leq 0.002$ on BA networks. The thick line is the mean field prediction for $h=0, \mathrm{Eq} \cdot(48)$.

In Fig 7 we check the scaling of the fire density $\eta \rho^{F}$ as a function of $\eta g / \ell$ in UCM networks with degree exponent $\gamma=1 / 2$. As in the case of the homogeneous WS networks, the data collapse is very good for large values of $\eta g / \ell$, fitting perfectly the theoretical prediction (full line) obtained by a numerical resolution of Eqs. (26) and (29). Again, deviations from the HMF prediction are observed for small $\eta g / \ell$, which must be attributed to the presence of strong dynamical correlations between vertices, which invalidate the HMF approximation.

The SF nature of this network model allows to explore the role of the degree in the establishment of dynamical correlations at small values of $\eta g / \ell$. In order to do so, we concentrate in this case on the conditional probabilities $P_{k}(\alpha \mid \beta)$ that a vertex of degree $k$ be in state $\alpha$, provided it is nearest neighbor of a vertex in state $\beta$. In absence of dynamical correlations, we should expect $P_{k}(\alpha \mid \beta) \equiv \rho_{k}^{\alpha}$ in the steady-state of the dynamics. In Fig. 8 we show
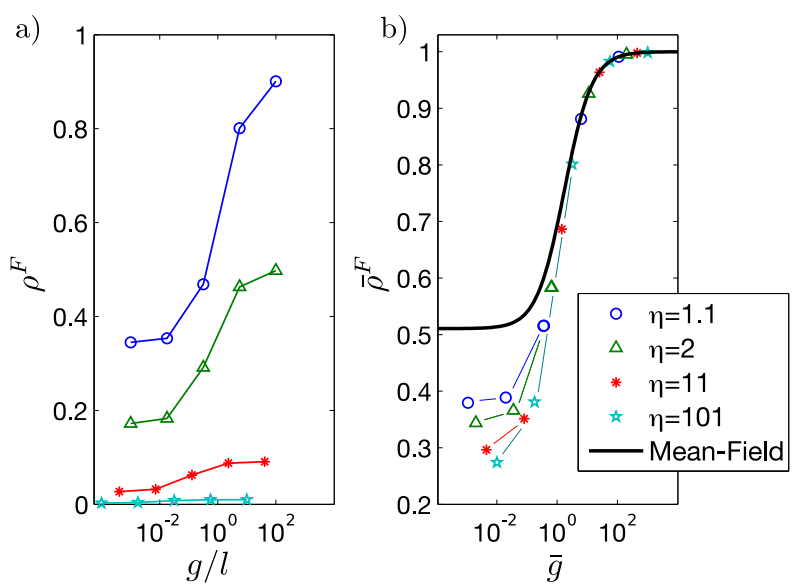

Fig. 7. Steady state fire density in the FFM on heterogeneous UCM networks with $\gamma=1 / 2$ and size $\mathrm{N}=10^{6}$. (a) Raw data for different values of $\eta$ as a function of $g / \ell$, and fixed $h / \ell=0.4$. (b) Data collapse of the previous plots as given in Eq.(14). The full line is a numerical solution to Eq. (26) plugged into (29) with the appropriate parameters $(m=2, \gamma=1 / 2, h / \ell=0.4)$. For small $\bar{g} \equiv \eta g / \ell$, deviations from the mean field theory are observed.

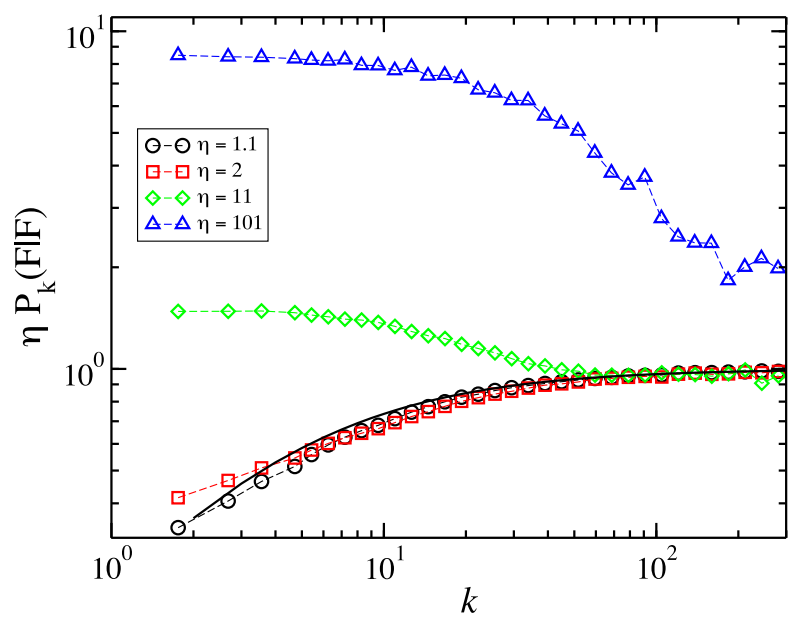

Fig. 8. Dependence of a vertex's state on his neighbors' states, as a function of the degree in UCM networks with $\gamma=\frac{1}{2}$ and size $N=10^{6}$. The thick line corresponds to the theoretical prediction $\bar{\rho}_{k}^{F}$, computed numerically from Eqs. (26) and (12). Data for $h / \ell=0.4, \bar{g}=0.01$.

the theoretical value $\bar{\rho}_{k}^{F}$ for $\bar{g}=10^{-2}$, calculated from Eqs.(26) and (12), compared with the conditional rescaled probability $\eta P_{k}(F \mid F)$, evaluated from numerical simulations and plotted as a function of $k$ for different values of $\eta$. In the absence of dynamical correlations, we should observe the plots of $\eta P_{k}(F \mid F)$ to collapse onto the theoretical curve $\bar{\rho}_{k}^{F}$ for the different values of $\eta$. While this scenario is correct for $\eta \leq 2$, we observe very strong deviations from the HMF prediction for large $\eta$, signaling the non-mean-field behavior of the FFM in SF networks. In particular, the conditional probability $P_{k}(F \mid F)$ turns out to be larger than the HMF average value $\rho_{k}^{F}=\eta^{-1} \bar{\rho}_{k}^{F}$, 
the difference increasing for small degree values. The discrepancy between $P_{k}(F \mid F)$ and the average fire density, also observed in homogeneous networks (see Fig. 8), is again due to the clustering at low fire densities of burning vertices in connected regions of the network.

\section{Conclusion}

In this paper we have presented a detailed analytical study of the forest fire model (FFM) in complex networks. From the perspective of the modelization of epidemic spreading processes, the FFM represents a generalization of several well-known epidemic models previously studied, which can be captured within the formalism of the FFM by the appropriate selection of representative parameters. Applying the now established HMF theory formalism, we have derived a set of rate equations in continuous time that represent the dynamics of this model. Focusing in the long term steady state behavior, we have defined a set of algebraic equations, whose analysis allows to discuss the role of the different parameters in the model. Thus, the rate at which empty sites become trees (the recovery rate of infected individuals) $p$, turns out to be absorbed in a rescaling of the fire density (density of infected individuals) and of the spontaneous ignition (spontaneous infection) rate, yielding in this way a fire density that is inversely proportional to $1 / p$. In the case of homogeneous networks, we recover the results previously obtained in the mean-field analysis of the FFM 39. In the case of heterogeneous SF networks, on the other hand, we have been able to provide exact explicit expressions for the fire density for a degree exponent $\gamma=1$, and approximate expressions for $\gamma<1$, valid for the cases of $h$ or $g$ very small.

A comparison of these theoretical predictions with large scale simulations in homogeneous and heterogeneous networks shows the HMF theory to provide a correct description of the steady state of the FFM for large $p$ (small $\eta$ ) and $g$, a regime in which the average fire density is sufficiently large. For small $p$ (large $\eta$ ) and $g$, on the other hand, numerical simulations indicate the breakdown of HMF theory. The origin of this failure can be traced back to the build up of dynamical correlations between nearest neighbor vertices, correlations which in fact are expected to appear, due to the fact that fires accumulate with large probability in connected clusters, and are therefore not homogeneously distributed over the network as assumed by mean-field approaches.

Apart from providing new insights into the behavior of a dynamical model relevant in epidemiological modeling, our results indicate a possible path to the understanding of the failure of HMF theory observed in other kinds of non-equilibrium processes in complex networks [7].

R.P.-S. acknowledges financial support from the Spanish MEC (FEDER) under Project No. FIS2007-66485-C02-01, and additional financial support through ICREA Academia, funded by the Generalitat de Catalunya. The authors thank M. Moret for making available to us the Bionics cluster, and C. Castellano for helpful comments and discussions.

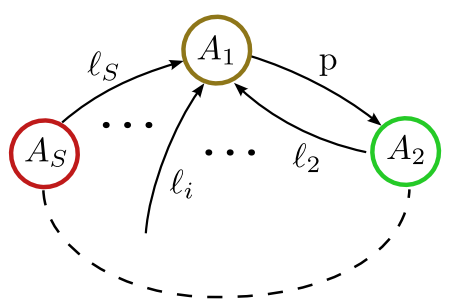

Fig. 9. General dynamical process with a rest state $A_{1}$.

\section{Appendix A: Rest states play no role in steady- state solutions}

Let us consider a general dynamical process taking place on $S$ states where $A_{1}$ is what we will call here a "rest state": every site $A_{i}$ can evolve to $A_{1}$ only with some fixed rate $\ell_{i}$ and $A_{1}$ evolves to $A_{2}$ with some other fixed rate $p$, see Fig. 9. The dynamical equations of this process take the general form:

$$
\left\{\begin{array}{rl}
\dot{a}_{1}(t) & =\sum_{i=2}^{S} \ell_{i} a_{i}(t)-p a_{1}(t) \\
\dot{a}_{2}(t) & =p a_{1}(t)+f_{2}\left[a_{2}(t), \ldots, a_{S}(t)\right]-\ell_{2} a_{2}(t) \\
& \vdots \\
\dot{a}_{i}(t) & =f_{i}\left[a_{2}(t), \ldots, a_{S}(t)\right]-\ell_{i} a_{i}(t) \\
& \vdots \\
\dot{a}_{S}(t) & =f_{S}\left[a_{2}(t), \ldots, a_{S}(t)\right]-\ell_{S} a_{S}(t) \\
1 & =\sum_{i=1}^{S} a_{i}
\end{array},\right.
$$

for some functions $f_{i}\left[a_{2}(t), \ldots, a_{S}(t)\right]$, where $a_{i}(t)$ is the probability of finding the system in state $i$ at time $t$. Trying to solve this system for the stationary state by setting $\dot{a}_{i}(t)=0 \forall i$, one can get rid of the $a_{1}$ variable by substituting its value from the first equation into the other ones, namely $a_{1}=\sum_{i} \ell_{i} / p a_{i}$. Then the last $S-1$ equations form a set of $S-1$ independent equations with only $S-1$ unknowns:

$$
\left\{\begin{array}{rl}
0 & =\sum_{i=2}^{S} \ell_{i} a_{i}+f_{2}\left(a_{2}, \ldots, a_{S}\right)-\ell_{2} a_{2}(t) \\
& \vdots \\
0 & =f_{i}\left(a_{2}, \ldots, a_{S}\right)-\ell_{i} a_{i}(t) \\
& \vdots \\
0 & =f_{S}\left(a_{2}, \ldots, a_{S}\right)-\ell a_{S} \\
1 & =\sum_{i=2}^{S} a_{i}+\sum_{i=2}^{S} \ell_{i} / p a_{i}
\end{array} .\right.
$$

Now if the introduced functions can be written as

$$
f_{i}\left(a_{2}, \ldots, a_{S}\right)=\sum_{n_{2}, \ldots, n_{S}} c_{i}^{n_{2}, \ldots, n_{S}} \cdot a_{2}^{n_{2}} \cdot \ldots \cdot a_{S}^{n_{S}},
$$


then, performing the change of variable

$$
\bar{a}_{i}=\eta_{i} a_{i}, \bar{c}_{i}^{n_{2}, \ldots, n_{S}}=c_{i}^{n_{2}, \ldots, n_{S}} \prod_{j} \eta_{j}^{-n_{j}}, \bar{\ell}_{i}=\ell_{i} \eta_{i}^{-1} \forall i
$$

keeps all the first equalities in (50) true for any value of the constants $\eta_{i}$. In particular, choosing

$$
\eta_{i}=1+\ell_{i} / p
$$

then all equalities are verified and the last one becomes $\sum_{i=2}^{S} \bar{a}_{i}=1$.

In other words, the new variables $\bar{a}_{i}$, together with Eqs. (50) above, describe the stationary solution of a dynamical system with analogous dynamics as the first one (49), up to a constant for some parameters, and without the rest state. Finding the stationary solution of the original $S$-states system is thus equivalent to finding the one for the new system with $S-1$ states.

The new model has one parameter less, $p$, which has been absorbed in the redefinition of all other parameters and variables of the new system. So the stationary state of the first model is somehow independent on $p$. This can be stated as:

$$
\begin{aligned}
& \eta_{i} a_{i}\left(\left\{\eta_{j}, \prod_{k} \eta_{k}^{n_{k}} \bar{c}_{j}^{n_{2}, \ldots, n_{S}}, \eta_{j} \bar{\ell}_{j}\right\}_{j}\right) \\
& \quad=\bar{a}_{i}\left(\left\{\bar{c}_{j}^{n_{2}, \ldots, n_{S}}, \bar{\ell}_{j}\right\}_{j}\right) \text { Indep. of } p \forall i
\end{aligned}
$$

Moreover, an upper bound for all densities $a_{i}$ can be deduced directly from this argument: Since $\bar{a}_{i}$ is the probability to be in state $i$ in the new system, then

$$
\bar{a}_{i}<1 \Rightarrow a_{i}<\eta_{i}^{-1}
$$

Notice that in the main text we chose the time scale so that $\bar{\ell}=1$. This has the effect of applying the $\eta$ factor to different coefficients (c.f. Eq. (10)).

\section{Appendix B: An explicit solution to a tran- scendental equation}

Let's consider the equation

$$
\kappa=a+b \kappa^{\gamma}
$$

for $\kappa$, where $a, b \in \mathbb{R}_{+}$are given and $0<\gamma<1$ is fixed. This equation was already considered back in 1772 by Lambert 48.

If $a \neq 0$ we can introduce the variable $v=\kappa / a$ and see that this new variable depends on a unique variable $\beta=b a^{\gamma-1}$ :

$$
v=1+\beta v^{\gamma} .
$$

Similarly, if $b \neq 0$, the new variable $u=\kappa b^{\frac{1}{\gamma-1}}$ only depends on $\alpha=a b^{\frac{1}{\gamma-1}}=\beta^{\frac{1}{\gamma-1}}$ :

$$
u=\alpha+u^{\gamma}
$$

So if we know how to express explicitly $v(\beta)$ or $u(\alpha)$, we can conversely do so with $\kappa(a, b)$. To achieve this, we use the Lagrange inversion theorem 49 which states that the reciprocal function $x(y)$ of $y=f(x)$ can be expressed around $y_{0}=f\left(x_{0}\right)$ as

$$
x=x_{0}+\sum_{n=1}^{\infty} c_{n}\left(y-y_{0}\right)^{n}
$$

with $c_{n}=\left.\frac{d^{n-1}}{d x^{n-1}}\left(\frac{x-x_{0}}{f(x)-y_{0}}\right)^{n}\right|_{x=x_{0}} \frac{1}{n !}$, if $f^{\prime}\left(x_{0}\right) \neq 0$.

(1) Case $a \neq 0$

In this case we have $\beta(v)=v^{-\gamma}(v-1)$ and so around $v_{0}=1$ and $\beta_{0}=0, \beta^{\prime}(1) \neq 0$ and we can calculate:

$$
\begin{aligned}
n ! c_{n} & =\left.\frac{d^{n-1}}{d v^{n-1}}\left(\frac{v-v_{0}}{\beta(v)-\beta_{0}}\right)^{n}\right|_{v=v_{0}} \\
& =\frac{\Gamma(n \gamma+1)}{\Gamma(n(\gamma-1)+2)}
\end{aligned}
$$

Thus we can write

$$
v(\beta)=1+\sum_{n=1}^{\infty} \frac{\Gamma(n \gamma+1)}{\Gamma(n(\gamma-1)+2) \Gamma(n+1)} \beta^{n} .
$$

This formulation of the function $v(\beta)$ is true as long as the series converges. To know what interval it corresponds to, we calculate the convergence radius $\beta_{c}$ following its definition:

$$
\begin{aligned}
\beta_{c}^{-1} & =\limsup _{n \rightarrow \infty} \sqrt[n]{\left|c_{n}\right|} \\
& =\limsup _{n \rightarrow \infty} \sqrt[n]{\left|\sin (n \pi(1-\gamma)) \frac{\Gamma(n \gamma+1) \Gamma(n(1-\gamma)-1)}{\Gamma(n+1)}\right|} \\
& =\lim _{n \rightarrow \infty} \sqrt[n]{\frac{\Gamma(n \gamma+1) \Gamma(n(1-\gamma)-1)}{\Gamma(n+1)}}
\end{aligned}
$$

where we have used the Euler reflection formula to avoid an undefined valued of the Gamma function 50. Using the Stirling formula $\Gamma(z)=\sqrt{\frac{w \pi}{z}}\left(\frac{z}{e}\right)^{z}\left(1+\mathcal{O}\left(\frac{1}{z}\right)\right)$ and $\lim _{n \rightarrow \infty} \sqrt[n]{n}=1$ we find

$$
\beta_{c}^{-1}=\gamma^{\gamma}(1-\gamma)^{1-\gamma}
$$

So Eq. (62) can be used to express $\kappa$ explicitly, but not for any value of its arguments.

(2) Case $b \neq 0$ :

Let's find now a series representation of function $u(\alpha)$. To simplify the calculation we introduce in this case $w=$ $u^{1-\gamma}$ so that

$$
w^{\frac{1}{1-\gamma}}=\alpha+w^{\frac{\gamma}{1-\gamma}} .
$$

We can now directly apply the same steps as before to find a power series expression for the reciprocal function of $\alpha(w)=w^{\frac{\gamma}{1-\gamma}}(w-1)$ around $w_{0}=1, \alpha=0$, since 
$\alpha^{\prime}(1) \neq 0:$

$$
\begin{aligned}
n ! c_{n} & =\left.\frac{d^{n-1}}{d w^{n-1}}\left(\frac{w-w_{0}}{\alpha(w)-\alpha_{0}}\right)^{n}\right|_{w=w_{0}} \\
& =\frac{\Gamma\left(-\frac{n \gamma}{1-\gamma}+1\right)}{\Gamma\left(-\frac{n}{1-\gamma}+2\right)} .
\end{aligned}
$$

Thus we have

$$
u(\alpha)=\left[1+\sum_{n=1}^{\infty} \frac{\Gamma\left(-\frac{n \gamma}{1-\gamma}+1\right)}{\Gamma\left(-\frac{n}{1-\gamma}+2\right) \Gamma(n+1)} \alpha^{n}\right]^{\frac{1}{1-\gamma}} .
$$

Again we can calculate the convergence radius and see that it is $\alpha_{c}=(1-\gamma) \gamma^{\frac{\gamma}{1-\gamma}}=\beta_{c}^{\frac{1}{1-\gamma}}$. In other words, we found a complete description of the solution $\kappa(a, b)$ of Eq. (56): depending on whether the value of $\beta$ is smaller or greater than $\beta_{c}$, development (62) or (70) converges and gives an explicit value for $\kappa(a, b)$ :

$\kappa(a, b)=\left\{\begin{array}{c}a \sum_{n=0}^{\infty} \frac{\Gamma(n \gamma+1)}{\Gamma(n(\gamma-1)+2) \Gamma(n+1)}\left(b a^{\gamma-1}\right)^{n} \\ \text { if } \beta<\beta_{c} \\ {\left[b\left(1-\sum_{n=1}^{\infty}(-1)^{n} \frac{\Gamma\left(\frac{n}{1-\gamma}-1\right)}{\Gamma\left(\frac{n \gamma}{1-\gamma}\right) \Gamma(n+1)}\left(a b^{\frac{1}{\gamma-1}}\right)^{n}\right)\right]^{\frac{1}{1-\gamma}}} \\ \text { if } \beta>\beta_{c}\end{array}\right.$

with

$$
\left.\beta_{c}=\gamma^{-\gamma}(1-\gamma)^{-(1-\gamma)} \in\right] 1,2[.
$$

Since $\kappa$ depends smoothly on $a$ and $b$, as can be seen from Eq. (56), the case $\beta=\beta_{c}$ is deducible as the limit of any of the two series.

This expression gives the value of the function $\kappa(a, b)$ whenever either $a$ or $b$ is different from zero. The solution in the case $a=b=0$ is trivially $x=0$.

\section{References}

1. A. Barrat, M. Barthélemy, A. Vespignani, Dynamical Processes on Complex Networks (Cambridge University Press, Cambridge, 2008)

2. S.N. Dorogovtsev, A.V. Goltsev, J.F.F. Mendes, Rev. Mod. Phys. 80, 1275 (2008)

3. A.L. Barabási, R. Albert, Science 286, 509 (1999)

4. G. Caldarelli, Scale-Free Networks: Complex Webs in $\mathrm{Na}$ ture and Technology (Oxford University Press, Oxford, 2007)

5. D.S. Callaway, M.E.J. Newman, S.H. Strogatz, D.J. Watts, Phys. Rev. Lett. 85, 5468 (2000)

6. R. Cohen, K. Erez, D. ben Avraham, S. Havlin, Phys. Rev. Lett. 86, 3682 (2001)

7. C. Castellano, R. Pastor-Satorras, Phys. Rev. Lett. 96, 038701 (2006)

8. K.I. Goh, D.S. Lee, B. Kahng, D. Kim, Phys. Rev. Lett. 91, 148701 (2003)
9. Y. Moreno, A. Vazquez, Europhys. Lett. 57, 765 (2002)

10. A. Arenas, A. Díaz-Guilera, J. Kurths, Y. Moreno, C. Zhou, Phys. Rep. 469, 93 (2008)

11. C. Castellano, S. Fortunato, V. Loreto, Rev. Mod. Phys. 81, 591 (2008)

12. M.J. Keeling, K.T.D. Eames, J. R. Soc. Interface 2, 295 (2005)

13. F. Liljeros, C.R. Edling, L.A.N. Amaral, H.E. Stanley, Y. Aberg, Nature 411, 907 (2001)

14. R. Pastor-Satorras, A. Vespignani, Phys. Rev. Lett. 86(14), 3200 (2001)

15. A.L. Lloyd, R.M. May, Science 292, 1316 (2001)

16. Y. Moreno, R. Pastor-Satorras, A. Vespignani, Eur. Phys. J. B 26, 521 (2002)

17. M.E.J. Newman, Phys. Rev. E 66, 016128 (2002)

18. R. Pastor-Satorras, A. Vespignani, Phys. Rev. E 65, 036104 (2001)

19. R. Cohen, S. Havlin, D. ben-Avraham, Physical Review Letters 91, 247901 (2003)

20. J.G. Gardenes, V. Latora, Y. Moreno, E. Profumo, Proc. Natl. Acad. Sci. USA 105, 1399 (2008)

21. V. Colizza, A.Barrat, M. Barthelemy, A. Vespignani, Proc. Natl. Acad. Sci. USA 103, 2015 (2006)

22. R.M. Anderson, R.M. May, Infectious diseases in humans (Oxford University Press, Oxford, 1992)

23. D.J. Watts, S.H. Strogatz, Nature 393, 440 (1998)

24. M. Boguñá, C. Castellano, R. Pastor-Satorras, Phys. Rev. E 79, 036110 (2009)

25. P. Bak, K. Chen, C. Tang, Phys. Lett. A 147, 297 (1992)

26. B. Drossel, F. Schwabl, Phys. Rev. Lett. 69, 1629 (1992)

27. P. Grassberger, New Journal of Physics 4, 17 (2002)

28. J.A. Bonachela, M.A. Muñoz, JSTAT p. P09009 (2009)

29. C. Rhodes, R. Anderson, Nature 381, 600 (1996)

30. G. Abramson, M. Kuperman, Phys. Rev. Lett. 86, 2909 (2001)

31. C.M. M. Müller-Linow, M.T. Hütt, Phys. Rev. E 74, 016112 (2006)

32. J. Liu, Y. Tang, Z. Yang, J. Stat. Mech. p. P08008 (2004)

33. S. Peng, Y. Li, B. Zheng, Steady states and critical behavior of epidemic spreading on complex networks (WCICA 2008. 7th World Congress on Intelligent Control and Automation, 2008), pp. 3481-3486

34. R. Pastor-Satorras, A. Vázquez, A. Vespignani, Phys. Rev. Lett. 87, 258701 (2001)

35. H.J. Jensen, Self-Organized Criticality (Cambridge University Press, Cambridge, 1998)

36. M. Boguñá, R. Pastor-Satorras, A. Vespignani, in Statistical Mechanics of Complex Networks, edited by R. PastorSatorras, J.M. Rubí, A. Díaz-Guilera (Springer Verlag, Berlin, 2003), Vol. 625 of Lecture Notes in Physics

37. R. Pastor-Satorras, A. Vespignani, Phys. Rev. E 63, 066117 (2001)

38. M. Catanzaro, M. Boguñá, R. Pastor-Satorras, Phys. Rev. E 71, 056104 (2005)

39. K. Christensen, H. Flyvbjerg, Z. Olami, Phys. Rev. Lett. 71, 2737 (1993)

40. J. Marro, R. Dickman, Nonequilibrium phase transitions in lattice models (Cambridge University Press, Cambridge, 1999)

41. M. Boguñá, R. Pastor-Satorras, Phys. Rev. E 66, 047104 (2002)

42. M. Abramowitz, I.A. Stegun, Handbook of mathematical functions. (Dover, New York, 1972) 
43. R.M. Corless, G.H. Gonnet, D.E.G. Hare, D.J. Jeffrey, D.E. Knuth, Advances in Computational Mathematics 5, $329(1996)$

44. A. Vázquez, R. Pastor-Satorras, A. Vespignani, Phys. Rev. E 65, 066130 (2002)

45. A. Barrat, R. Pastor-Satorras, Phys. Rev. E 71, 036127 (2005)

46. M. Catanzaro, M. Boguná, R. Pastor-Satorras, Phys. Rev. E 71, 027103 (2005)

47. M. Boguñá, R. Pastor-Satorras, A. Vespignani, Euro. Phys. J. B 38, 205 (2004)

48. J.H. Lambert, Nouveaux mémoires de l'Académie royale des sciences et belles-lettres $\mathbf{1}$ (1772)

49. E. Goursat, Course in Mathematical Analysis, Vol. 2: Functions of a Complex Variable 8 Differential Equations (Dover, New York, 1959)

50. J. Havil, Exploring Euler's Constant (Princeton University Press, Princeton, NJ, 2003) 